\title{
Adherence to the EAU guidelines on Penile Cancer Treatment: European, multicentre, retrospective study
}

\author{
Maida Bada ${ }^{1}$ (1) - Francesco Berardinelli ${ }^{1}$ - Peter Nyiràdy ${ }^{2}$. Judith Varga ${ }^{2} \cdot$ Pasquale Ditonno $^{3} \cdot$ Michele Battaglia $^{3}$. \\ Paolo Chiodini ${ }^{4}$. Cosimo De Nunzio ${ }^{5}$ Giorgia Tema ${ }^{5}$ - Alessandro Veccia ${ }^{6}$. Alessandro Antonelli ${ }^{6}$ Luca Cindolo $^{1}$. \\ Claudio Simeone $^{6} \cdot$ Stefano Puliatti $^{7} \cdot$ Salvatore Micali ${ }^{7} \cdot$ Luigi Schips $^{8}$
}

Received: 14 June 2018 / Accepted: 13 February 2019

○) Springer-Verlag GmbH Germany, part of Springer Nature 2019

\begin{abstract}
Purpose The European Association of Urology (EAU) guidelines for penile cancer (PC) are exclusively based on retrospective studies and have low grades of recommendation. The aim of this study was to assess the adherence to guidelines by investigating the management strategies for primary tumours and inguinal lymph nodes.

Methods We retrospectively reviewed the clinical charts of $176 \mathrm{PC}$ patients who underwent surgery in eight European centres from 2010 to 2016. The stage and grade were assessed according to the 2009 AJCC-UICC TNM classification system. To assess adherence rates, we compared theoretical and practical adherence to the EAU guidelines.

Results Overall, 176 patients were enrolled. Partial amputation was the most frequent surgical approach (39\%). 53.7\% of tumours were stage Tis-T1b and the remaining $46.3 \%$ were stage T2-T4. Palpable lymph nodes were detected in $30.1 \%$ of patients and $45.1 \%$ underwent lymphadenectomy (LY). A sizeable group of tumours (43.2\%) were N0. For primary treatment, adherence to the EAU guidelines was good (66\%). In non-adherent cases, reasons for discrepancy were patient's choice $(17 \%)$, surgeon's preference (36\%), and other causes (47\%). For LY, the guideline adherence was $70 \%$, with either patient's or surgeon's choice or other causes accounting for discrepancy in 28,20 , and $52 \%$ of non-adherent cases, respectively.

Conclusion Adherence to the EAU guidelines for PC was quite high across the eight European centres involved in the study. This notwithstanding, strategies for further improvement should be developed and evenly adopted.
\end{abstract}

Keywords Penile cancer $\cdot$ Adherence $\cdot$ EAU guidelines $\cdot$ Lymphadenectomy $\cdot$ Partial amputation $\cdot$ Total amputation

Maida Bada

maida.bada@yahoo.com

1 Department of Urology, S. Pio da Pietrelcina Hospital, ASL 2 Abruzzo, Vasto, Italy

2 Department of Urology, Hospital of Budapest, Budapest, Hungary

3 Department of Emergency and Organ Transplantation, Urology and Andrology Unit II, University of Bari, Bari, Italy

4 Department of Medical Statistics Unit, Second University, Naples, Italy

5 Department of Urology, Sant'Andrea Hospital, Sapienza University, Rome, Italy

6 Department of Urology, Spedali Civili Hospital, Brescia, Italy

7 Department of Urology, Baggiovara Hospital, University of Modena and Reggio Emilia, Modena, Italy

8 Department of Urology, G. D’Annunzio University, Chieti, Italy

\section{Introduction}

Penile cancer (PC) is a rare malignancy and a potentially mutilating disease for men. In the United States and Western countries, it accounts for $0.4-0.6 \%$ of all malignancies (Clark et al. 2013). The major risk factor for $P C$ is an uncircumcised penis (Maden et al. 1993). Other factors include HPV infection, smoke, and low socio-economic status (Rubin et al. 2001; Harish and Ravi 1995; Daling et al. 2005). About 95\% of penile cancers are squamous cell carcinomas (SCC) and can develop anywhere on the penis (Christodoulidou et al. 2015). Most commonly, these tumours appear as a lump or nodule (47\%), an ulcer (35\%), an erythematous lesion (17\%), or an incidental finding at circumcision $(0.7 \%)$ (Hernandez et al. 2008). If treated at an early stage, $P C$ has a cure rate of $80 \%$. Pathologic stage or grade of the primary tumour and subsequent locoregional lymph node metastasis drive survival, with node-positive patients having 5-year survival 
rates ranging from 20 to $80 \%$ (Pandey et al. 2006). The optimal treatment for primary tumour depends on lesion's size, location, and possibility of oncological radicality preserving both quality of life and sexual satisfaction. Since PC is a rare disease, the treatment is not always uniform and often depends on surgeon's experience and patient's preferences. For example, radical inguinal lymph node dissection (ILND) is the standard of care for node-positive patients and is potentially curative in half of the cases (McDougal 2005); however, its complexity and associated high degree of morbidity (wound healing and lymphoedema) often discourage use (Thuret et al. 2011). Theoretically, guidelines should provide evidence-based guidance to physicians and patients alike. Although following established guidelines has been found to improve patient outcomes and enhance consistency of practices, some studies on solid cancer treatment report extremely variable adherence rates, ranging from 40 to $99 \%$ (Heins et al. 2016). The aim of this study was to evaluate adherence to the EAU guidelines in a multicentre series of patients surgically treated for penile cancer.

\section{Materials and methods}

We retrospectively reviewed the clinical charts of 176 PC patients who underwent surgery in 8 European centres from 2010 to 2016. The study was approved by the Ethics Committee of each participating institution and all the procedures were performed in accordance with the Declaration of Helsinki. Information on demographics, patients' comorbidities, circumcision, site of primary lesion, local therapy, lymph node management, and histopathological data was collected and analysed. Staging was as per the 2009 AJCC-UICC TNM classification system and was performed with a combination of physical examination and CT and/or MRI. Treatment of the primary tumour and the decision to proceed to inguinal lymph node dissection were established according to local protocols based on the 2009 EAU guidelines. For patients with penile carcinoma in situ (CIS), the European Association of Urology recommends topical chemotherapy with 5\% fluorouracil and imiquimod (Alnajjar et al. 2012). For $\mathrm{Ta} / \mathrm{T} 1$ lesions, laser ablation is a valid treatment (Colecchia et al. 2009). For penile glans tumours, glans resurfacing and glansectomy are preferred (Pizzocaro et al. 2010). When tumours extend into the corporeal bodies, urethra, and adjacent structures (T2-T4), partial penectomy provides excellent oncologic control (Opjordsmoen and Fossa 1994). When a negative margin cannot be achieved, or a large fungating tumour is present, total amputation with perineal urethrostomy is recommended (Garaffa et al. 2009). As regards inguinal lymph node management, patients with clinically node-negative (cN0) pTis, pTa, and pT1a tumours are at low risk of inguinal metastasis and are suitable candidates for observation. For patients with intermediate-to high-risk tumours (pT1b, T2-T4), either modified inguinal lymph node dissection (ILND) or dynamic sentinel node biopsy (DSLNB) is recommended (Hughes et al. 2010). Patients with clinically positive lymph nodes $(\mathrm{cN} 1 / \mathrm{cN} 2)$ are at high risk of metastatic disease and radical ILND is in order. Enlarged fixed inguinal lymph nodes (cN3) require multimodal treatment by chemotherapy followed by radical ILND in clinically responsive cases (Pizzocaro et al. 2010). In our study, indications for pelvic lymph node dissection were $>2$ proven inguinal metastases, involvement of the femoral (Cloquet's) node, and radiological suspicion of pelvic lymph node involvement. Follow-up was by a combination of physical examination and imaging based on risk stratification and was updated by recall to the clinic. Theoretical adherence to the EAU guidelines for primary surgery and lymphadenectomy was assessed for each case. A case was considered adherent when the surgical approach was perfectly aligned with the guidelines. For non-adherent cases, reasons for discrepancy were analysed and grouped as follows: (1) patient's choice; (2) surgeon's preference; and (3) other. Statistical analysis was performed using SPSS (version 25.0). Data evaluation confirmed a non-normal distribution of the study dataset. Intergroup differences in medians for quantitative variables were tested using the Kruskal-Wallis oneway analysis of variance, and differences in distributions for categorical variables were calculated with a Chi square test. Using multiple logistic regression with the ENTER method, the statistically significant age-adjusted variables as assessed by univariate analysis were entered and investigated as predictors of adherence to the EAU guidelines. The threshold for significance was set at alpha $=0.05$. Data are presented as mean \pm standard deviation (SD). Odds ratios and $95 \%$ confidence interval (CI) were calculated for the parameters in each group using "non-adherence" as the reference group.

\section{Results}

Patients' demographics, surgical details, and histopathological findings are reported in Table 1. Overall, 176 patients were enrolled (mean age $66 \pm 11.3$ ). All patients had histopathologically confirmed squamous cell carcinoma of the penis. Tumours were mainly located at the glans (55\%), 38\% were stage pT1, and $83 \%$ had negative surgical margins. Penile partial amputation was the most common surgical approach (39\%). At diagnosis, $30.1 \%$ of patients had palpable inguinal lymph nodes. ILND was performed in $45.1 \%$ of patients $(15 \% \mathrm{cN} 0$ and $80 \% \mathrm{cN} 1 / \mathrm{cN} 2$; the remaining $5 \%$, although meeting the EAU criteria, declined surgery). Nodal status was available for 74 patients: 32 patients were $\mathrm{pN} 0,19$ were $\mathrm{pN} 1$, and 23 were pN2. For primary treatment, adherence to the EAU guidelines was good (66\%) (Fig. 1). In 


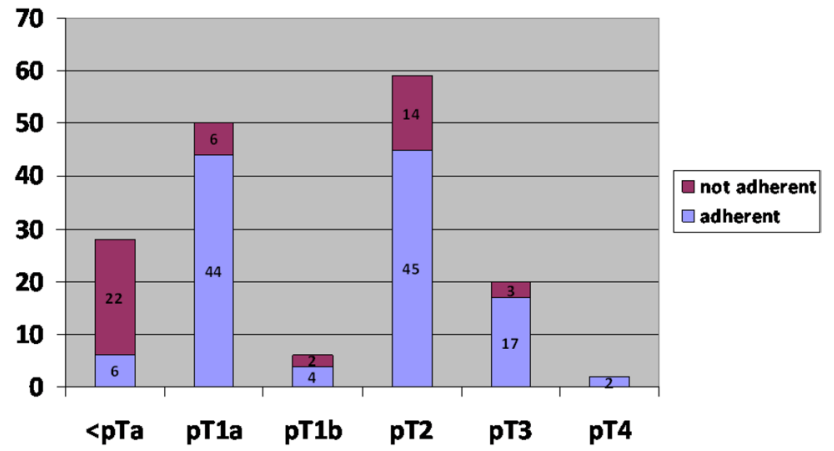

Fig. 1 Adherence distribution based on pT stages

non-adherent cases, reasons for discrepancy were patient's choice (17\%), surgeon's preference (36\%), and other causes (47\%) (Table 2). For lymph node treatment, guideline adherence was $70 \%$ (Fig. 2), with either patient's or surgeon's choice or other causes accounting for discrepancy in 28, 20, and $25 \%$ of non-adherent cases, respectively (Table 2). Univariate analysis showed no significant differences between adherent and non-adherent patients, whereas at multivariate analysis, the stage appeared to be the only predictor of adherence for primary treatment (OR 2.61, 95\% CI 1.34-5.06, $p=0.005$ ) (Table 3). At a mean follow-up of 2 years, 126 patients were alive, 35 had died from cancer progression, and the remaining 15 had died of other causes.

\section{Discussion}

Clinical guidelines provide scientifically informed guidance to both physicians and patients for making appropriate health care decisions. They have the potential to improve outcomes, enhance consistency of practices across institutions, and support quality improvement activities (Heins et al. 2016; Catalona 1988). The European Association of Urology constantly produces and updates guidelines covering all fields of urology. The EAU guidelines on PC were first published in 2001 and received a major update in 2009, concurrently with the release of a new version of the TNM Classification of Malignant Tumors (TNM). Although most urologists are familiar with the EAU recommendations, adherence may be poor for several reasons. First of all, penile cancer is a rare disease in industrialised countries, which means that the approved guidelines are mostly based on small, methodologically weak studies (e.g., retrospective studies, opinion letters, case reports etc.). As a consequence, they will have a low level of evidence and degree of recommendation. Numerous factors may influence adherence in clinical practice. Although treatment of the primary tumour can be curative for PC, guideline recommendations are not always followed, especially when a generally poor prognosis and a
Table 1 Patients' demographics: clinical, surgical and pathological characteristics $(N=176)$

\begin{tabular}{|c|c|c|}
\hline & $N$ & $\%$ \\
\hline Age, mean (SD) & $66.5(11.3)$ & - \\
\hline \multicolumn{3}{|l|}{ Comorbidities } \\
\hline Diabetes & 40 & 22.7 \\
\hline Hypertension & 70 & 39.7 \\
\hline Cardiovascular disease & 16 & 9 \\
\hline Dyslipidaemia & 8 & 4.5 \\
\hline Multiple comorbidities & 22 & 12.5 \\
\hline No comorbidities & 20 & 11.6 \\
\hline \multicolumn{3}{|l|}{ Circumcision (14 missing) } \\
\hline Yes & 70 & 43.5 \\
\hline No & 92 & 56.5 \\
\hline \multicolumn{3}{|l|}{ Site of lesion } \\
\hline Prepuce & 20 & 10.8 \\
\hline Glans & 96 & 54.9 \\
\hline Both & 60 & 34.3 \\
\hline Size of lesion [mean (SD)] & $3(1.8)$ & \\
\hline \multicolumn{3}{|l|}{ Palpable lymph nodes } \\
\hline No & 123 & 69.9 \\
\hline Yes & 53 & 30.1 \\
\hline \multicolumn{3}{|l|}{ Histologic findings } \\
\hline \multicolumn{3}{|l|}{ pTNM } \\
\hline Tis-T1a & 94 & 53.7 \\
\hline $\mathrm{T} 1 \mathrm{~b}-\mathrm{T} 4$ & 81 & 46.3 \\
\hline \multicolumn{3}{|l|}{ Grading } \\
\hline G1 & 65 & 37.1 \\
\hline $\mathrm{G} 2$ & 83 & 46.9 \\
\hline G3 & 28 & 16 \\
\hline \multicolumn{3}{|l|}{ Vascular invasion } \\
\hline No & 138 & 78.9 \\
\hline Yes & 38 & 21.1 \\
\hline \multicolumn{3}{|l|}{ Lymphovascular invasion } \\
\hline No & 140 & 79.4 \\
\hline Yes & 36 & 20.6 \\
\hline \multicolumn{3}{|l|}{ Surgical margins } \\
\hline $\mathrm{R} 0$ & 147 & 83.4 \\
\hline $\mathrm{R} 1$ & 29 & 16.6 \\
\hline \multicolumn{3}{|l|}{ Nodal status } \\
\hline pNO & 32 & 43.2 \\
\hline $\mathrm{pN} 1$ & 19 & 25.7 \\
\hline $\mathrm{pN} 2$ & 23 & 31.1 \\
\hline \multicolumn{3}{|l|}{ Type of surgery } \\
\hline Circumcision & 12 & 6,9 \\
\hline Local excision & 42 & 24 \\
\hline Glansectomy & 27 & 15.3 \\
\hline Partial penectomy & 69 & 38.9 \\
\hline Total penectomy & 26 & 14.9 \\
\hline \multicolumn{3}{|l|}{ Lymphadenectomy } \\
\hline No & 96 & 54.9 \\
\hline Yes & 80 & 45.1 \\
\hline
\end{tabular}


Table 2 Adherence rates for primary treatment and lymphadenectomy and reasons for non-adherence

\begin{tabular}{lccccc}
\hline & \multicolumn{2}{c}{$\begin{array}{l}\text { Adherence (primary } \\
\text { treatment) }\end{array}$} & & \multicolumn{2}{c}{$\begin{array}{l}\text { Adherence (lym- } \\
\text { phadenectomy) }\end{array}$} \\
\cline { 2 - 3 } & $N$ & & & & $\%$ \\
\hline Yes & 116 & 65.7 & & 122 & 69.7 \\
No & 60 & 34.3 & & 54 & 30.3 \\
Surgeon's choice & 22 & 35.6 & & 11 & 20 \\
Patient's choice & 10 & 17 & & 16 & 28 \\
Other causes & 28 & 47.5 & & 27 & 52 \\
\hline
\end{tabular}

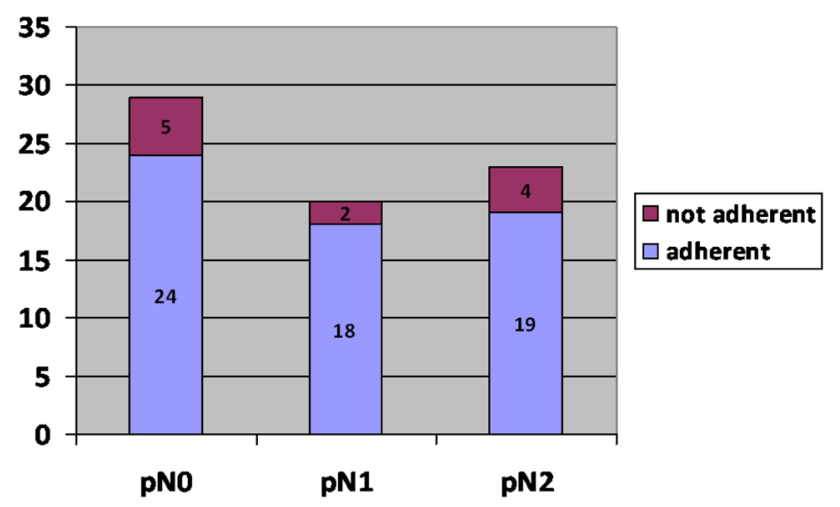

Fig. 2 Adherence distribution based on $\mathrm{pN}$ stages

high rate of serious side effects are expected (Leone et al. 2017; Pizzocaro and L Piva 1988; Breen et al. 2015). For example, physicians may be less likely to adhere to established treatment recommendations when patients have severe comorbidities or poor general health. In our study, treatment of the primary tumour was in line with the guidelines in $66 \%$ of cases, with stage being the only significant predictor of adherence (OR 2.61, 95\% CI 1.34-5.06, $p=0.005$ ). Not surprisingly, physicians are more likely to follow procedures with which they are more familiar. Nowadays, PC is not a topic of frequent discussion in clinical practice (Cabana et al. 1999). It is also an emotive disease and both under- and overtreatment can have considerable short- and long-term repercussions (Kumar et al. 2012). The patient is actively involved in the clinical decision-making process, perhaps even more so than for any other kind of tumour given the potentially devastating effects on quality of life and mental well-being. Our data confirm that the wider discrepancy between guidelines and clinical practice is typical of localised tumours that in theory should be better treated with organ-preserving techniques. It is worth noting that there currently is no widespread definition of penile sparing surgery. Historically, treatment was by radical or partial amputation with a 2-cm margin for oncologic efficacy. Over time, guidelines began to favour a more conservative approach whenever possible. Local recurrence has a minimal impact on long-term survival. Horenblas et al.'s multivariate analysis demonstrated no difference in cancer-specific survival for patients treated with penile sparing surgery vs. partial amputation (Djajadiningrat et al. 2004). Despite current trends, some less experienced centres continue to have an all-too radical approach which is not in line with the latest guidelines. A more extensive experience in treating PC would doubtlessly allow for the safer use of conservative techniques.

Our study was not geared to describe reasons for nonadherence in detail, but individual choice appears to be an important factor for both patients (24\%) and physicians (34\%), the latter often mentioning lack of training and skill as the reason for departing from recommendations. In the remaining $42 \%$ of cases, we were unable to establish the exact reasons for non-compliance. The EAU guidelines recommend ILND in cN0 patients with pT1b and T2-T4 tumours, as well as in all $\mathrm{cN} 1 / \mathrm{cN} 2$ patients. Despite lymph node status being the primary determinant of survival in penile carcinoma (D'Ancona et al. 2004), only a fraction of patients who are candidates for ILND undergo the procedure. Our results indicate an overall ILND rate of $45.1 \%$,
Table 3 Population's characteristics based on the 2016 EAU guidelines; adherence rates for primary treatment

\begin{tabular}{lccc}
\hline & $\begin{array}{l}\text { Non-adherent patients } \\
(60 / 176) 35 \%\end{array}$ & $\begin{array}{l}\text { Adherent patients } \\
(115 / 176) 65 \%\end{array}$ & $p$ \\
\hline Age (years) [mean (SD)] & $68.3(11.2)$ & $65.5(11.2)$ & 0.113 \\
Size (cm) [mean (SD)] & $3(2.3)$ & $3(1.65)$ & 0.387 \\
Circumcision (\%) (missing =16) & $21 / 53(13.1 \%)$ & $48 / 107(30 \%)$ & 0.324 \\
PT stage (\%) & & & \\
Tis-T1a & $41 / 60(23.4 \%)$ & $53 / 115(30.3 \%)$ & 0.004 \\
T1a-T4 & $19 / 60(10.9 \%)$ & $62 / 115(35.4 \%)$ & \\
PN stage (\%) & & & \\
N0 & $8 / 17(10.8 \%)$ & $24 / 57(32.4 \%)$ & 0.741 \\
N1 & $5 / 17(6.8 \%)$ & $14 / 57(18.9 \%)$ & \\
N2 & $4 / 17(5.4 \%)$ & $19 / 57(25.7 \%)$ & \\
\hline
\end{tabular}


a figure consistent with previous estimates (ILND ranges 39.1-43.6\%, for all SCC stages) (Ficarra et al. 2005; Ornellas et al. 2008). Adherence to the EAU ILND guidelines was $70 \%$ in our study. This is surprisingly high, even admitting that clinical practice should strive for maximum correspondence with international recommendations. The result is also in sharp contrast with the findings by Leijte et al., whose analysis of compliance with the NCI ILND guidelines reported adherence rates of only $27.6 \%$ (Leijte et al. 2008). Non-adherence may be ascribed to lack of surgical experience or insufficient familiarity with inguinal lymphadenectomy. Practical constraints may also have a part, for example, in the non-ubiquitous availability of sentinel node biopsies.

Our study is not devoid of limitations. First, its retrospective design limits the quality of the data. Second, a central pathology review could have contributed to higher accuracy of pathologically assessed variables, and hence the lack of it may represent another weakness. However, none of the previously reported multi-institutional studies relied on central pathology (Leijte et al. 2008). Third, complications and length of hospitalisation could not be analysed because the database was not designed to take these parameters into account. Fourth, the small number of patients limited deep survival analysis, which is important for future research. Finally, our study cohort originates from eight European centres, each with its own definition of disease progression and varying levels of surgical skills. Despite this obvious limitation, all multicentre studies benefit from greater generalisability of results, which increases proportionally to the number of surgeons and clinical sites involved.

\section{Conclusion}

Adherence to the EAU guidelines for PC was quite good, both for treatment of the primary tumour and for ILND. New strategies for further improvement may be key to ensuring better care in terms of quality and efficacy. These include specific surgical training and the formation of super-regional networks with a multidisciplinary approach that will likely favour high-volume centres.

\section{Compliance with ethical standards}

Conflict of interest The authors declare that they have no conflict of interest.

Research involving human participants and/or animals This article does not contain any studies with human participants or animals performed by any of the authors.

Informed consent For this type of study formal consent is not required.

\section{References}

Alnajjar HM, Lam W, Bolgeri M, Rees RW, Perry MJ, Watkin NA (2012) Treatment of carcinoma in situ of the glans penis with topical chemotherapy agents. Eur Urol 62(5):923-928

Breen KJ, O'Connor KM, Power DG, Mayer NJ, Rogers E, Sweeney P (2015) Penile cancer-Guideline adherence produces optimum results. Surgeon 13(4):200-206

Cabana MD, Rand CS, Powe NR et al (1999) Why do not physicians follow clinical practice guidelines? A framework for improvement. JAMA 282:1458-1465

Catalona WJ (1988) Modified inguinal lymphadenectomy for carcinoma of the penis with preservation of saphenous veins: technique and preliminary results. J Urol 140:306-310

Christodoulidou M, Sahdev V, Houssein S, Muneer A (2015) Epidemiology of penile cancer. Curr Probl Cancer 39(3):126-136

Clark PE, Spiess PE, Agarwal N et al (2013) National comprehensive cancer network. Penile cancer: clinical practice guidelines in oncology. J Natl Compr Cancer Netw 11:594-615

Colecchia M, Nicolai N, Secchi P et al (2009) pT1 penile squamous cell carcinoma: a clinicopathologic study of 56 cases treated by $\mathrm{CO} 2$ laser therapy. Anal Quant Cytol Histol 31(3): 153-160

D'Ancona CAL, de Lucena RG, Querne FA, de O, Martins, Denardi MHT, Netto F NR (2004) Long-term follow up of penile carcinoma treated with penectomy and bilateral modified inguinal lymphadenectomy. J Urol 172(2):498-501

Daling JR, Madeleine MM, Johnson LG et al (2005) Penile cancer:importance of circumcision, human papillomavirus and smoking in in situ and invasive disease. Int $\mathrm{J}$ Cancer 116(4):606-616

Djajadiningrat RS, van Werkhoven E, Horenblas S (2015) Prophylactic pelvic lymph node dissection in patients with penile cancer. $\mathbf{J}$ Urol. 193:1976

Ficarra V, Zattoni F, Cunico CSC et al (2005) Lymphatic and vascular embolizations are independent predictive variables of inguinal lymph node involvement in patients with squamous cell carcinoma of the penis. Cancer 103:2507-2516

Garaffa G, Raheem AA, Christopher NA, Ralph DJ (2009) Total phallic reconstruction after penile amputation for carcinoma. BJU Int 104:852-856

Harish K, Ravi R (1995) The role of tobacco in penile carcinoma. Br J Urol 75(3):375-377

Heins MJ, de Jong JD, Spronk I, Ho VK, Brink M, Korevaar JC (2016) Adherence to cancer treatment guidelines: influence of general and cancer-specific guideline characteristics. Eur J Public Health 27:616-620

Hernandez BY, Barnholtz-Sloan J, German RR et al (2008) Burden of invasive squamous cell carcinoma of the penis in the United States. Cancer 113(10 Suppl):2883-2891

Hughes BE, Leijte JA, Kroon BK et al (2010) Lymph node metastasis in intermediate-risk penile squamous cell cancer: a two-centre experience. Eur Urol 57:688-692

Kumar P, Singh S, Goddard JC, Terry TR, Summerton DJ (2012) The development of a supraregional network for the management of penile cancer. Ann R Coll Surg Engl 94(3):204-209

Leijte JAP, Kirrander P, Antonini N, Windahl T, Horenblas S (2008) Recurrence patterns of squamous cell carcinoma of the penis: recommendations for follow-up based on a two-centre analysis of 700 patients. Eur Urol 54:161-168

Leone A, Diorio GJ, Pettaway C, Master V, Spiess PE (2017) Contemporary management of patients with penile cancer and lymph node metastasis. Nat Rev Urol 14(6):335-347

Maden C, Sherman KJ, Beckmann AM et al (1993) History of circumcision, medical conditions, and sexual activity and risk of penile cancer. J Natl Cancer Inst 85:19-24 
McDougal WS (2005) Preemptive lymphadenectomy markedly improves survival in patients with cancer of the penis who harbor occult metastases. J Urol 173:681

Opjordsmoen S, Fossa SD (1994) Quality of life in patients treated for penile cancer. A follow-up study. Br J Urol 74:652-657

Ornellas AA, Kinchin EW, Nobrega BL, Wisnescky A, Koifman N, Quirino R (2008) Surgical treatment of invasive squamous carcinoma of the penis: Brazilian National Cancer Institute long-term experience. J Surg Oncol 97:487-495

Pandey D, Mahajan V, Kannan RR (2006) Prognostic factors in nodepositive carcinoma of the penis. J Surg Oncol 93(2):133-138

Pizzocaro G, L Piva (1988) Adjuvant and neoadjuvant vincristine, bleomycin, and methotrexate for inguinal metastases from squamous cell carcinoma of the penis. Acta Oncol 27:823-824

Pizzocaro G, Algaba F, Horenblas S et al (2010) European Association of Urology (EAU) Guidelines Group on Penile Cancer. EAU penile cancer guidelines 2009. Eur Urol 57(6):1002-1012
Rubin MA, Kleter B, Zhou M et al (2001) Detection and typing of human papillomavirus DNA in penile carcinoma: evidence for multiple independent pathways of penile carcinogenesis. Am J Pathol 159(4):1211-1218

Thuret R, Sun M, Lughezzani G et al (2011) A contemporary population- based assessment of the rate of lymphnode dissection for penile carcinoma. Ann Surg Oncol 18:439-446

Publisher's Note Springer Nature remains neutral with regard to jurisdictional claims in published maps and institutional affiliations. 